\title{
ON THE ZEROS OF A CONFLUENT HYPERGEOMETRIC FUNCTION ${ }^{1}$
}

JET WIMP

1. Introduction. In this note, by using an integral representation due to Erdélyi, we prove a theorem concerning the zeros of the confluent hypergeometric function $\Psi(a, c \mid z)$. As a corollary, we prove that the zeros of the Bessel polynomials for $\delta \geqq 0, n \geqq 1$, lie in the lefthalf plane.

\section{Fundamental theorems.}

THEOREM. Let $a$ and $c$ be real and $2 a-c>-1$. Then any zero $z_{p}$ of $\Psi(a, c \mid z)$ must satisfy $\operatorname{Re}\left(z_{\nu}\right)<0$.

Proof. In the formula [2, p. 287, equation (21)]

$$
\begin{aligned}
& \Gamma(\gamma) \Psi(a, c \mid x) \Psi\left(a^{\prime}, c \mid y\right) \\
& \quad=\int_{0}^{\infty} e^{-t} t^{-1}(x+t)^{-a}(y+t)^{-a_{2}}{ }_{2} F_{1}\left(\begin{array}{c}
a, a^{\prime} \\
\gamma
\end{array} \mid \frac{t(x+y+t)}{(x+t)(y+t)}\right) d t, \\
& \gamma=a+a^{\prime}-c+1, \quad \operatorname{Re}(\gamma)>0, x y \neq 0,
\end{aligned}
$$

let $x=\sigma+i \tau=z, y=\sigma-i \tau=\bar{z}, a=a^{\prime}, \gamma=2 a-c+1$. Then

$$
\begin{aligned}
& \Psi(a, c \mid z) \Psi(a, c \mid \bar{z}) \\
& \quad=\int_{0}^{\infty} \frac{e^{-t} t^{\gamma-1}\left[(t+\sigma)^{2}+\tau^{2}\right]^{-a}}{\Gamma(\gamma)}{ }_{2} F_{1}\left(\begin{array}{c}
a, a \\
\gamma
\end{array} \mid \frac{t(t+2 \sigma)}{(t+\sigma)^{2}+\tau^{2}}\right) d t .
\end{aligned}
$$

Since $\gamma>0$, the integrand of (2) is a series of strictly positive terms. Thus we have

$$
\Psi(a, c \mid z) \Psi(a, c \mid \bar{z})>0, \quad \operatorname{Re}(z) \geqq 0, z \neq 0 .
$$

A known result [6] excludes the point $z=0$ from consideration, and the proof of the theorem is complete.

We now define the Bessel polynomials by

Received by the editors September 9, 1963 and, in revised form, October 11, 1963.

1 This research was sponsored by the United States Air Force through the Aeronautical Research Laboratories, Office of Aerospace Research, under Contracts AF 33(616)-7062 and AF 33(657)-8872. The author is indebted to the referee for his suggestions. 


$$
P_{n}^{(\delta)}(z)=\sum_{k=0}^{n}\left(\begin{array}{l}
n \\
k
\end{array}\right)(n+\delta)_{k} z^{n-k}, \quad \delta \geqq 0, n \geqq 1,
$$

where

$$
(n+\delta)_{k}=(n+\delta)(n+\delta+1) \cdots(n+\delta+k-1),
$$

$$
(n+\delta)_{0}=1 \text {. }
$$

For a treatment of these polynomials and specializations, see, for example, [1], [3], [4].

McCarthy [5] has proved that for $n$ fixed and $\delta$ sufficiently large, the zeros of (4) are in the left-half plane. Applying our theorem, we may prove a more general result.

Corollary. Every zero $z_{\nu}{ }^{(n)}$ of (4) satisfies $\operatorname{Re}\left(z_{\nu}{ }^{(n)}\right)<0$.

Proof. Consider the function

$$
f(z)=\Psi(-n,-2 n-\delta+1 \mid z) .
$$

We also have

$$
f(z)=z^{2 n+\delta} \Psi(n+\delta, 2 n+\delta+1 \mid z) .
$$

We distinguish two cases. $^{2}$ First let $\delta$ be nonintegral. Then by $[2$, p. 257, equation (7)] and (6),

$$
\begin{aligned}
f(z) & =(n+\delta) \sum_{k=0}^{n}\left(\begin{array}{l}
n \\
k
\end{array}\right) \frac{(-z)^{k}}{(-2 n-\delta+1)_{k}} \\
& =P_{n}^{(\delta)}(z),
\end{aligned}
$$

as can be seen by turning the sum around. Now let $\delta$ be integral and use (7) and [2, p. 261, equation (13)].

$$
\begin{aligned}
f(z) & =(n+\delta)_{n} \sum_{k=0}^{2 n+\delta-1}\left(\begin{array}{l}
n \\
k
\end{array}\right) \frac{(-z)^{k}}{(-2 n-\delta+1)_{k}}, \\
& =P_{n}^{(\delta)}(z) .
\end{aligned}
$$

Our theorem furnishes the desired result for $\delta>0$, with $a=-n$, $c=-2 n-\delta+1$, and $\gamma=\delta$. Also, we may let $\delta \rightarrow 0$ and the integral (2) in the limit exists, because

2 This is necessary because $\Psi(a, c \mid x)$ assumes two different forms depending on whether or not $c$ is an integer. Note also (4) defines a polynomial of degree $n, n \geqq 0$, $\delta \neq-1,-2, \cdots$, but we prefer to define $P_{n}^{(\delta)}(z)$ so the corollary holds. 


$$
\begin{aligned}
f(z) f(\bar{z})= & \lim _{\delta \rightarrow 0} \int_{0}^{\infty} \frac{e^{-t} t^{\delta-1}}{\Gamma(\delta)}\left[(t+\sigma)^{2}+\tau^{2}\right]^{n} d t \\
& +\int_{0}^{\infty} e^{-t}\left[\left(t^{2}+\sigma^{\sigma} \sigma\right)^{2}+\tau^{2}\right]^{n} \sum_{k=1}^{n} \frac{t^{k-1}(-n)_{k}^{2}}{k ! \Gamma(k)} \\
& \cdot\left[\frac{(t+2 \sigma)}{(t+\sigma)^{2}+\tau^{2}}\right]^{k} d t \\
= & \left(\sigma^{2}+\tau^{2}\right)^{n}+\int_{0}^{\infty} e^{-t}\left[(t+\sigma)^{2}+\tau^{2}\right]^{n} \sum_{k=1}^{n} \frac{t^{k-1}(-n)_{k}^{2}}{k ! \Gamma(k)} \\
& \cdot\left[\frac{(t+2 \sigma)}{(t+\sigma)^{2}+\tau^{2}}\right]^{k} d t .
\end{aligned}
$$

Thus the same argument applies in the limiting case and the proof of the corollary is complete.

Added in proof. We have noted that Buchholz (Die konfluente hypergeometrische Funktion, p. 90, Springer, Berlin, 1953) proves a similar theorem (excluding $z=0$ ) for the related function $W_{k, m}(z)$.

\section{REFERENCES}

1. W. A. Al-Salam, The Bessel polynomials, Duke Math. J. 24 (1957), 529-545.

2. A. Erdélyi, W. Magnus, F. Oberhettinger and F. G. Tricomi, Higher transcendental functions, Vol. I, McGraw-Hill, New York, 1953.

3. Emil Grosswald, On some algebraic properties of the Bessel polynomials, Trans. Amer. Math. Soc. 71 (1951), 197-210.

4. H. C. Krall and Orrin Frink, A new class of orthogonal polynomials, the Bessel polynomials, Trans. Amer. Math. Soc. 65 (1949), 100-115.

5. P. J. McCarthy, Approximate location of the zeros of generalized Bessel polynomials, Quart. J. Math. Oxford Ser. (2) 12 (1961), 265-267.

6. L. J. Slater, Confluent hypergeometric functions, Cambridge Univ. Press, Cambridge, 1960; p. 105, (6.1.2).

Midwest Research Institute, Kansas City, Missouri 